\title{
高抗马铃薯纺锤形块茎类病毒的转基因 马铃薯细胞内核酶转录产物的分布
}

刘灿辉杨希才王小风田波 (中国科学院微生物研究所. 北京 100080 )

摘要 以对马铃薯纺锤形块茎类病毒 (PSTVd) 有高度抗性的表达核酶的转基因马铃薯为材 料, 研究了核酶转录产物在植株根细胞中的亚细胞分布. 首先通过往返式凝胶电泳和 Northern 杂交选择出表达核酶并对 PSTVd 高抗的, 即检测不出PSTVd 存在的马铃薯系. 用其根 细胞进行原位杂交, 结果表明核酶转录产物主要分布在转基因马铃薯细胞的细胞核中. 这一 结果支持我们提出的核酶对在核内复制的类病毒更为有效的假说.

\section{关键词 转基因马铃薯 PSTVd 原位杂交 核酶亚细胞分布}

核酶是能专一性切割 RNA 的小 RNA 分子. 具有抑制病毒基因表达的巨大潜力. 核酶 对病毒 RNA 在体外切割试验中显示出抑制作用. 但体内对细胞质内复制的病毒 RNA 的抑 制则不明显 ${ }^{[1 ~ 3]}$, 例如转基因烟草中只表达低水平的切割烟草花叶病毒( TMV) RNA 的核 酶. TMV 感染后, 它们的病毒症状得到延缓. 但不能阻止 T M V 的复制和发病 ${ }^{[4,5]}$.

最近, 我们发现表达切割 PSTVd 负链 RNA 的核酶转基因马铃薯对 PST Vd 有高度抗 性 $^{[6]}$; 还证明表达切割花椰菜花叶病毒( CaMV) 35S RNA 核酶转基因油菜对 CaM V 的感染 有强抗性 ${ }^{[7]}$. 有趣的是, 前者完全在核内复制, 后者有一核内复制阶段. 基于核酶对切割细 胞质内复制的病毒 RNA 和核内复制阶段的病毒 RNA 不同的抗性效应, 我们曾提出在转基因 情况下, 核酶对有核内复制阶段的类病毒和病毒更为有效的论点 ${ }^{[6]}$. 为了验证这一假设, 我 们应用原位杂交技术分析了对 PST Vd 复制有高抗性的转基因马铃薯中核酶的亚细胞分布. 本文报道核酶的转录产物主要分布在转基因马铃薯细胞核内的结果, 为上述假设提供了有力 证据.

\section{1 材料与方法}

( ) 试验材料. 转基因马铃薯 (Solanum tuberosum cultiva Desiree) 是转化了切割 PSTVd 负链核酶 ( Rz $)$ 的转基因植株, 对 PSTVd 的感染表现出强的抗性. 该核酶切割 PSTVd 负链 RNA 端区的 GUC/ G 位点( 324 321), 它的 5 端臂和 3 端臂各有 10 个和 11 个核苷酸的识别 序列. 以空载体转化植株作为对照 ${ }^{6]}$. 马铃薯种植于 $20 \sim 25{ }^{\circ} \mathrm{C}$ 的温室中, 所有马铃薯苗在 长至 7 8 片叶片阶段时接种 $20 \mathrm{ng}$ 的 PSTVd.

（）往返式凝胶电泳和 Northern 杂交. 总 RNA 用往返式聚丙烯酰胺凝胶电泳后进行

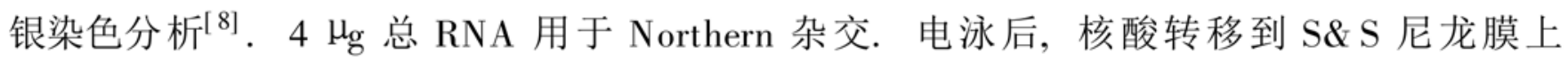
(Schleicher \& Schuell). 尼龙膜在 $80{ }^{\circ} \mathrm{C}$ 处理 $2 \mathrm{~h}$, 用 ${ }^{32} \mathrm{P}$ 标记的 PST Vd cDNA 或核酶 cDNA 进 行 Northern 杂交. 预杂交、杂交和放射自显影按文献所述进行 ${ }^{[6]} \cdot{ }^{32} \mathrm{P}$ 标记的 DNA 探针以凝 胶纯化的 PSTVd 克隆或核酶 $\mathrm{cDNA}$ 克隆的限制性片段作为模板, 通过寡核苷酸引物延伸法 制备而成. 
( ) 制备马铃薯根尖组织切片. $\mathrm{Rz}(-)$ 转化的和空载体转化的新鲜马铃薯苗在 FAA 固 定液( $50 \%$ 乙醇, $10 \%$ 福尔马林, $5 \%$ 乙酸) 中室温固定 $12 \mathrm{~h}$. 弃掉固定液，根尖用 PBS 洗 2 次, 置于冰冻切片机标本固定头上, 进行 $8 \mu_{\mathrm{m}}$ 切片; 附于涂有 $100 \mu_{\mathrm{g} / \mathrm{mL}}$ 多聚赖氨酸的载 片上，每 1 张玻片放 2 3 张切片，空气干燥，然后在 $1 \mu_{\mathrm{g}} / \mathrm{mL}$ 蛋白酶 $\mathrm{K}$ 中 $37^{\circ} \mathrm{C} \mathrm{\eta}_{\mathrm{q}}$ 育 $15 \mathrm{~min}$, 最后在含 $2 \mathrm{mg} / \mathrm{mL}$ 的甘氨酸的 PBS 中洗 4 次 ${ }^{[9]}$.

( ) 制备 ${ }^{35} \mathrm{~S}$ 标记的核酶 cDNA 探针. 以 5 CAGGAGGTTTCGTCC $3^{\prime}$ 和 5 TGTGGT TCACACCT $3^{\prime}$ 为引物, 以核酶基因双体为模板 ${ }^{[7]}$, 应用 PCR 方法制备 ${ }^{35} \mathrm{~S}$ 标记的核酶 $\mathrm{cD}^{-}$ $\mathrm{NA}$ 探针 $(80 \mathrm{bp})$. $100 \mu \mathrm{L}$ 的反应混合液包括: 2 个引物各 $100 \mathrm{pmol}, 1 \mathrm{ng}$ 核酶基因, dCTP,

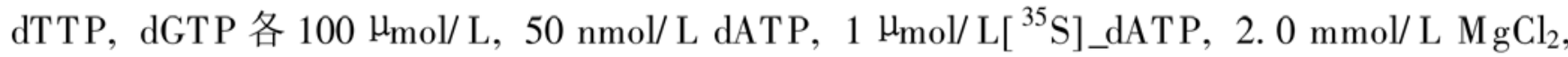
$50 \mathrm{mmol} / \mathrm{L} \mathrm{KCl}, 0.1 \mathrm{~g} / \mathrm{L}$ gelatin, $10 \mathrm{mmol} / \mathrm{L} \mathrm{Tris} \cdot \mathrm{HCl}(\mathrm{pH}=8.3)$ 和 $2.5 \mathrm{U}$ 的 $\mathrm{T}$ aq 酶. 反应 混合液上覆盖 $50 \mu \mathrm{L}$ 的石蜡油. PCR 反应进行 30 个循环, 反应条件为 $94{ }^{\circ} \mathrm{C}$ 变性 $30 \mathrm{~s}, 40{ }^{\circ} \mathrm{C}$ 退火 $30 \mathrm{~s}, 72{ }^{\circ} \mathrm{C}$ 延伸 $30 \mathrm{~s}$, 末次循环后, 再 $72{ }^{\circ} \mathrm{C}$ 延伸 $10 \mathrm{~min} .{ }^{35} \mathrm{~S}$ 标记探针的比放射性活性为 2 $\times 10^{8} / \mathrm{min} \cdot \mu_{\mathrm{g}}$.

（）原位杂交. 玻片在不含探针的杂交缓冲液中 $37{ }^{\circ} \mathrm{C}$ 预杂交 $2 \mathrm{~h}$. 杂交缓冲液包括: $50 \%$ 去离子甲酰胺，2 $\times$ SSC，10\% 硫酸葡聚糖，5 × Denhardt' s, $100 \mathrm{H}_{\mathrm{g}} / \mathrm{mL}$ 变性的鲑精 $\mathrm{DNA}, 100 \mu \mathrm{g} / \mathrm{mL}$ 酵母 $\mathrm{tRNA}, 100 \mathrm{mmol} / \mathrm{L} \mathrm{DTT}^{[10.111}$. 然后弃掉预杂交液, 每张切片加入 $10 \mu \mathrm{L}$ 含 $0.5 \mathrm{ng} / \mathrm{mL}$ 变性的 ${ }^{35} \mathrm{~S}$ 标记的核酶 $\mathrm{cDNA}$ 探针的杂交液. 加硅化盖玻片于杂交液上, 边缘用橡胶水泥 (rubber cement) 封闭, 以防止杂交液蒸发, 玻片在密闭湿盒中, $37^{\circ} \mathrm{C}$ 保温 16 $20 \mathrm{~h}$. 将玻片从湿盒取出, 去掉橡胶水泥, 于含 $50 \%$ 甲酰胺的 $2 \times \mathrm{SSC}$ 中 $37{ }^{\circ} \mathrm{C}$ 洗 $30 \mathrm{~min}$, 于含 $50 \%$ 甲酰胺的 $1 \times \mathrm{SSC}$ 中洗 $30 \mathrm{~min}$, 然后于 $1 \times \mathrm{SSC}$ 中洗 $30 \mathrm{~min}$. 于 $70 \%, 90 \%, 100 \%$ 乙醇梯度脱水, 空气干燥.

( ) 检测 ${ }^{35} \mathrm{~S}$ 标记的杂交体. 在暗室中, 将玻片浸入与水 1: 1 混合的核_4 乳胶( 中国原 子能研究所制造), 缓慢取出, 水平放置于玻片架上, 室温干燥 3 $4 \mathrm{~h}$, 将包被乳胶的玻片置 于暗盒中 $4{ }^{\circ} \mathrm{C}$ 曝光 $3 \sim 5 \mathrm{~d}$. 曝光后于 D_19 显影液中 $20{ }^{\circ} \mathrm{C}$ 显影 $2 \sim 4 \mathrm{~min}$, 蒸馏水冲洗 2 次, 然 后于 F_5 定影液中定影 $8 \mathrm{~min}$. 定影后, 玻片用 $10 \% \mathrm{Giemsa}$ 染 $2.5 \mathrm{~min}$, 于光学显微镜下观 察结果.

\section{2 结果}

\section{1 高抗 PSTVd 的核酶转基因植株品系的获得}

Northern 杂交表明: PST Vd 接种 1 个月后，一些核酶转基因马铃薯系( TR7 和 TR8) 检测 不到 PST Vd, 说明核酶能有效抵抗 PSTVd 的复制. 相反, 空载体转化植株中(图 1(a)、(b)), PST Vd 的复制照常进行. 某些转基因马铃薯系( TR6 和 TR9) 与对照相比, PST V d 的复制有 所减弱. 另外, 在所有没有 PSTVd 积累的抗病马铃薯系中, 都检测到核酶转录产物(图 1 (b) ), 表明核酶的转录正常进行. 更重要的是, 在 TR7 和 TR8 的后代中, 均检测不到 PSTVd RNA(图 1(c)), 被选为进行原位杂交试验材料.

\section{2 核酶转录产物主要定位于高抗 PSTVd 转基因马铃薯细胞核中}

为了定位核酶转录产物, 将接种后几乎没有 PSTVd 复制的转基因系 TR7 和 TR8 进行了 核酶原位杂交. 应用 TR7P1_3 和 TR8P1_3 各 3 个独立的无性繁殖的子代(图 1(c)). 在转基 因马铃薯根尖的细胞核中, 我们发现有大量的信号, 而在细胞质中只检测到少量信号(图 2 


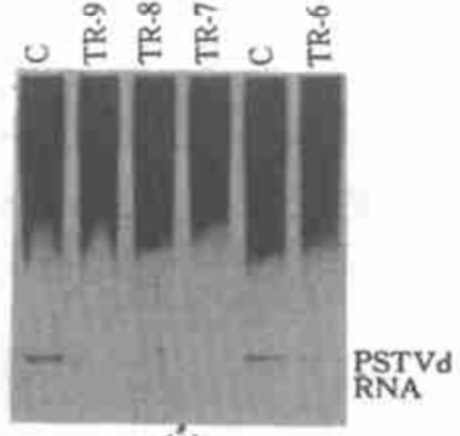

(a)

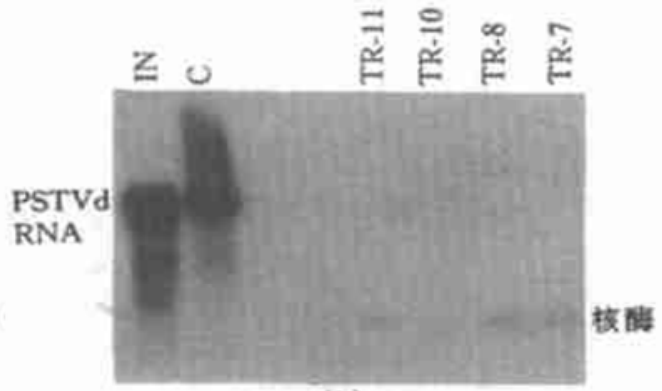

(b)

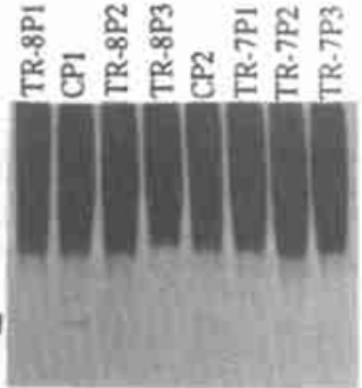

(c)

图 1 在转基因马铃薯植株中核酶的表达及对 PST Vd 复制的抗性

(a) 接种 PST Vd 的转基因马铃暮中 PST Vd 的检测. C 一一空载体 pROK_ef转化的植株, TR6 9 为 pROK_(-) Rz $(-$ ) 转化的植株; (b) Northern 杂交分析从转基因马铃暮中分离的 RNA. IN 一一接种 $0.1 \mu_{\mathrm{g}}$ PST Vd 的植株, C一空载 体 pROK_国转化的植株, TR7 11 一转基因植株的核酶转录产物; (c) 接种 PSTVd 的无性繁殖的子代植株中

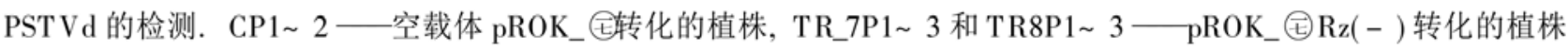

(a)、(c)) ; 在空载体转化植株的细胞中几乎检测不到什么信号(图 2(b)). 我们对根尖切片的 400 个细胞的银颗粒数进行了统计. 在转基因系的每一细胞中发现平均约有 90 个银颗粒, 在 空载体转化系中平均有 10 个银颗粒( 表 1), 这显然是非特异性杂交信号. 我们发现超过一半 的信号分布在仅占细胞一小部分的核中(表 1, 图 2(a)、(c)). 这些结果表明核酶转录产物主 要分布在核中.

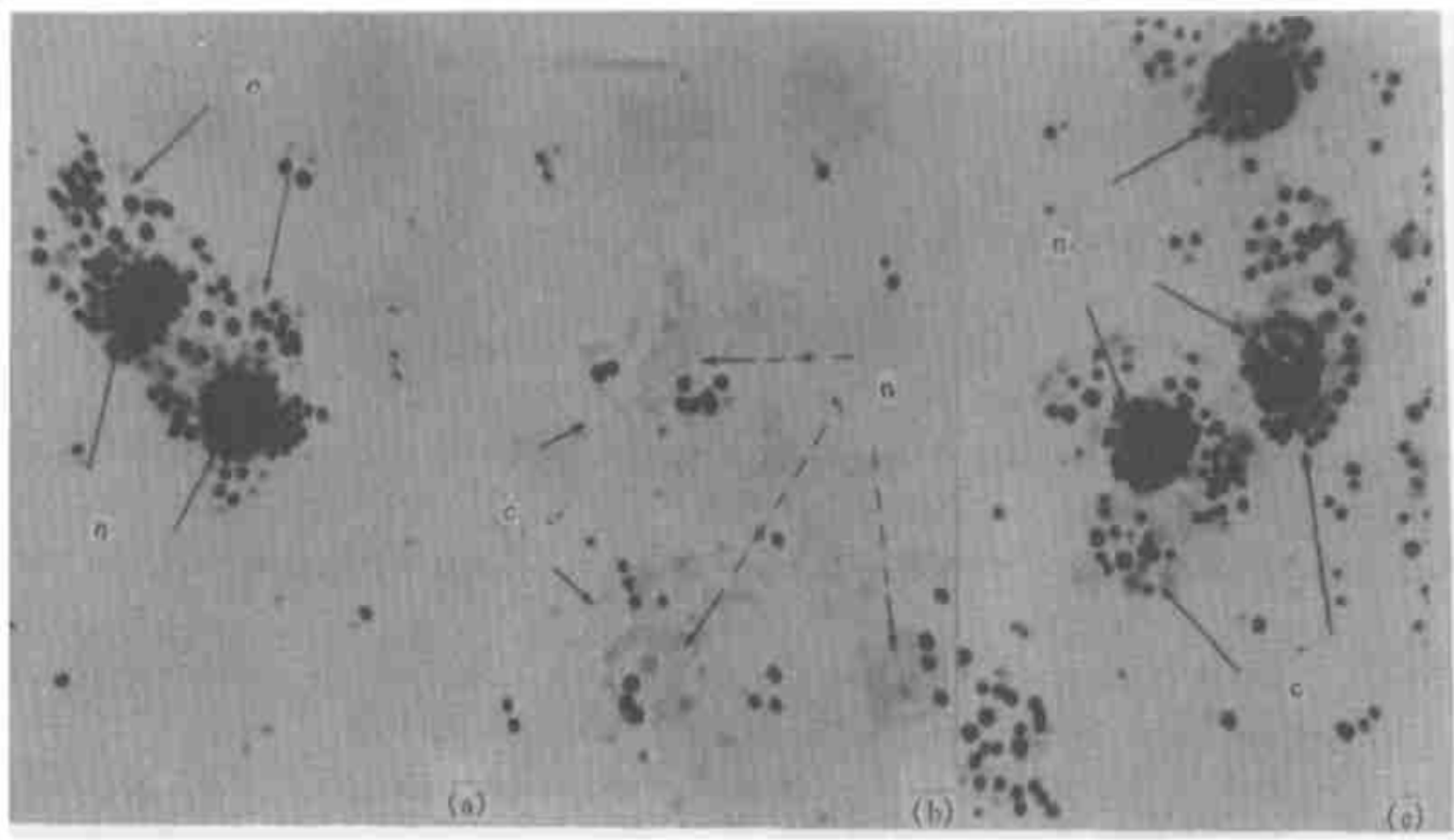

图 2 原位杂交检测转基因马铃薯苗根尖细胞中的核酶分子

（a）转基因马铃薯 TR7 根尖细胞, (b) 空载体转化的对照株细胞, (c) 转基因马铃薯 TR8 根尖细胞. $\mathrm{c}$ 一一细胞质, $\mathrm{n}$ 一细胞核. 黑点代表来自于杂交而显影的银颗粒 
表 1 原位杂交检测核酶在转基因马铃薯根尖细胞中的亚细胞分布

\begin{tabular}{|c|c|c|c|c|}
\hline \multirow{2}{*}{ 测试材料 } & \multicolumn{3}{|c|}{ 平均银颗粒数 } & \multirow{2}{*}{$\frac{\text { 核 }}{\text { 细胞 }} \times 100 / \%$} \\
\hline & 细胞 & 核 & 质 & \\
\hline 空载体转化的细胞 & $10 \pm 1.5$ & $2 \pm 1.0$ & $8 \pm 2.1$ & 20 \\
\hline $\mathrm{Rz}(-)$ 转化系 TR7P1 3 的细胞 ${ }^{a)}$ & $87.5 \pm 9.4$ & $51.0 \pm 10.6$ & $36.5 \pm 8.6$ & 58.3 \\
\hline $\mathrm{Rz}(-)$ 转化系 TR8P1 3 的细胞 ${ }^{\mathrm{b})}$ & $91.3 \pm 13.7$ & $53.9 \pm 12.5$ & $37.4 \pm 7.2$ & 59.0 \\
\hline
\end{tabular}

a) 在 $4 \times 10^{2}$ 个被观察的细胞中 $3.8 \times 10^{2}$ 个细胞是银颗粒阳性细胞, b) 在 $4 \times 10^{2}$ 个被观察的细胞中 $3.9 \times 10^{2}$ 个细胞 是银颗粒阳性细胞

\section{3 讨论}

我们在前文中提出核酶对只在核中复制的类病毒或有核复制阶段的病毒比在细胞质中复 制的病毒可能更为有效的论点 ${ }^{[6]}$. 本文提供了一个重要的证据来支持这一假设. 原位杂交 表明，大多数的核酶转录产物定位于核中，并且核的体积远小于细胞质，因此核酶在核中分 布的相对浓度远比在细胞中的高，这与它对核内复制的病毒 RNA 或类病毒 RNA 的有效切割 是一致的.

植物通过染色体转基因的情况下，核酶基因的转录产物首先出现在细胞核中，这可能是 核酶对完全在核内复制的 PST Vd 能够高度抑制其复制，达到用现有方法不能检测出 PST Vd 存在的原因. 对 PSTVd 失活的核酶对其复制没有抑制作用 ${ }^{[6]}$. 而对只在核内有复制阶段的 CaM V 只有中度抑制其复制的作用，因为失活的核酶作为一反义 RNA，也对 CaMV 的复制有 低度抑制 ${ }^{[7]}$. 为了有效切割在细胞质中复制的病毒, 将核酶基因插入到在细胞质中复制和积 累的病毒载体是可以尝试的一个途径.

致谢＼cjkstart作者感谢薛勇彪博士和庄蔚华小姐在准备本文中给予的帮助，感谢丁明孝教授、周海英老师和吴世安 同学给予的技术帮助.

\section{参 考 文 献}

1 Sarver N, Cantin E M, Chang P S, et al. Ribozyme as potential anti_HIV_1 therapeutic agents. Science, 1990, 247: $1222 \sim 1225$

2 Ojwang J O, Hampel A, Looney D J, et al. Inhibition of human immunodeficiency virus type 1 expression by a hairpin ri bozyme. Proc Natl Acad Sci USA, 1992, 89: 10 802 10806

3 Xing Z, Whitton J L. Ribozyme which cleave arenavirus RNAs: identification of susceptible target sites and inhibition by target site secondary structure. J Virol, 1992, 66: $1301 \sim 1309$

4 Edington B V, Pixon R A, Nelson R S. Ribozyme: description and uses. In: Hiatt D ed. Transgenic Plants, New York: Academic Press Inc, 1992. 301 323

5 De Feyter R, Young M, Schroeder K, et al. A ribozyme gene and an antisense gene are equally effective in conferring resistance to tobacco mosaic virus on transgenic tobacco. Mol Gen Genet, 1996, 250: 329 338

6 Yang X C, Yie Y, Zhu F, et al. Ribozyme_mediated high resistance against potato spindle tuber viroid in transgenic potatoes. Proc Natl Acad Sci USA, 1997, 94: 4 861 4865

7 王苏燕, 叶 寅, 赵淑珍, 等. 转基因油菜中核酸介导的对花梆菜花叶病毒的高度抗病性. 中国科学, C 辑, 1997, 27( 5) : $426 \sim 431$

8 Schumacher J, Meyer N, Riesnor D, et al. Diagnostic procedure for detection of viroids and viruses with circular RNAs by “return”_gel electrophoresis. J Phytopathol, 1986, 115: 332 343

9 Langdale J A. In situ hybridization. In: Freeling M, Walbot V, eds. The Maize Handbook, New York: Springer Verlag 
Inc, 1994. 165 179

10 Berger C N. In situ hybridization of immunoglobulin_specific RNA in single cells of the B lymphocyte lineage with radiolabelled DNA probes. EMBO J, 1986, 5: 85 93

11 Sideras P, Funa K. , Zalcberg_Quintana I, et al. Analysis by in situ hybridization of cells expressing mRNA for interleukin 4 in the developing thymus and in peripheral lymphocytes from mice. Proc Natl Acad Sci USA, 1988, 85: 218 221

\section{用整体原位杂交法研究微管蛋白基因 在斑马鱼胚胎中的表达}

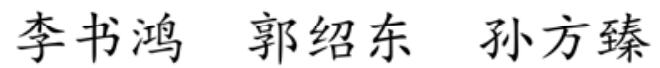

(中国科学院发育生物学研究所, 北京 100080)

摘要 斑马鱼是一个很好的脊椎动物发育研究模型, 斑马鱼胚胎发育过程中基因表达调控的 研究对揭示脊椎动物形态发生的分子机理非常重要. 微管蛋白固定受精卵细胞质中的形态发 生决定子, 组织形成基本的细胞骨架, 控制细胞的各种运动. 在脊椎动物中已发现有几种微管 蛋白, 每种微管蛋白都有其特定的表达位点和功能. 本研究用一种 $\beta_{2}$ 微管蛋白基因的全长 $\mathrm{cDNA}$ 为模板, 合成地高辛标记的 RNA 为探针, 对斑马鱼各时期的胚胎进行整体原位杂交. $\beta_{2}$ 微管蛋白基因的 mRNA 最初出现于斑马鱼囊胚中期胚胎的特定区域, 随着发育的进行, 这 种 mRNA 逐渐局限于斑马鱼胚胎的神经系统中. 这些结果表明, $\beta_{2}$ 微管蛋白基因在斑马鱼胚 胎中的表达是神经系统特异的.

\section{关键词＼cjkstart微管蛋白基因 整体原位杂交 斑马鱼}

神经发生( neurogenesis) 是动物形态发生过程中最早也是最重要的阶段之一, 神经发生过 程中一些重要基因表达调控的研究对揭示形态发生的分子机理非常重要. Richter 等人 ${ }^{[1]}$ 用 差异篮选法分离到了一个爪蟾胚胎发育基因, 通过 Northern 杂交显示该基因在爪蟾原肠胚、 神经胚、蝌蚪及成体的脑部表达. 后经测序知道该基因是一种 $\beta_{2}$ 微管蛋白基因 ${ }^{[2]}$. Oschw ald 等人 ${ }^{[3]}$ 通过整体原位杂交(whole mount in situ hybridization) 表明该基因的表达最早出现在爪 蟾囊胚晚期胚胎中; 从神经胚开始, 该基因的表达位点仅限于胚胎的脑和脊索. 斑马鱼是很好 的脊椎动物发育牛物学研究模型 ${ }^{[4,5]}$. 研穷微管蛋白的同源基因在斑马鱼肧胎发育中的时空 表达有着重要意义. 我们以爪蟾这个神经系统特异的微管蛋白基因的全长 cDNA 为模板, 体 外合成地高辛(Digoxygenin) 标记的反义 RNA, 用它作探针对斑马鱼胚胎发育各个阶段的胚胎 和孵化后的幼鱼进行了整体原位杂交. 本实验的目的有两个, 一是建立斑马鱼整体原位杂交 技术, 二是研究该微管蛋白基因在斑马鱼胚胎中的表达模式. 本实验结果显示, $\beta_{2}$ 微管蛋白 基因的同源基因在斑马鱼胚胎发育过程中从囊胚中期开始表达, 其表达位点从动物极观察时 呈马蹄型(图 1(a)). 随着神经系统的形成, 该基因的表达逐渐局限于神经系统, 捊化后的幼 鱼仅在脑部检测到该基因的表达产物. 本实验的结果表明: (1) 我们已经成功地建立了斑马 鱼整体原位杂交技术; (2) $\beta_{2}$ 微管蛋白基因的同源基因在斑马鱼胚胎发育过程中的表达是神 